\title{
How to Describe and Measure Obstacles of Narrative Immersion in a Film?
}

\author{
The Wheel of Immersion as a Framework
}

\author{
Thomas Bjørner, Andreas Magnusson, Robin Pascal Nielsen
}

\begin{abstract}
The aim of this study is to describe and measure obstacles of narrative immersion in a film. Inspired by a literature review within both game research and film studies, we propose a circular model to describe the dynamic process of different levels of involvement viewers can be in while watching a film. The evaluation is based on a 3D animation short film we have developed to achieve total immersion among viewers. The methodological design involved an attempt to decrease viewers' involvement in the animation film by using distractions during the viewing. The study follows a mixed method strategy combining observation, a questionnaire and a structured interview. The results revealed that viewers react very differently to the distractions. For some viewers, the animation film was not the perceptual focus, where others were totally immersed. The number of distractions was not dependent on whether the film was watched individually or in groups, and for all participants, the distractions occurred in certain rhythms.
\end{abstract}

Keywords: immersion, narrative, emotion, short film, mixed methods, 3D

\section{Introduction}

Immersion is used in many different contexts and refers to very different understandings, even within the same field. We are going to use the term immersion within the context of watching a 3D animation film. Most people have experienced how films have the ability to draw them in. This could be seen as reflecting shared emotions with the characters or whether viewers are affected by the film through evoked emotions (Hemenover \& Schimmack 2007; Tan 1996). The experience of total immersion occurs when viewers focus almost all of their attention on the film, and when it is so involving that they do not notice things around them, such as the amount of time that has passed or another person calling their name (Jennett et. al. 2008).

A literature review of the research on immersion shows that the majority of studies concern interactivity in computer games and virtual reality (Brown \& Cairns 2004; Calleja 2011; Ermi \& Mäyrä 2005; Jennett et al. 2008; Murray et al. 2007; Qin et al. 2009; Ryan 2001). The literature within cinematic immersion is rather scarce, so in the present study we would like to focus on immersive experiences in a $3 \mathrm{D}$ animation short film that concentrates on the narrative. There are studies focusing on technological advances and how they are related to immersed experiences (Recuber 2007; Nedelcu 
2013). There are other studies indicating that the extent of immersion is determined by the narrative influences of the story (Brown \& Cairns 2004; Busselle \& Bilandzic 2009; Douglas \& Hargadon 2001; Gross \& Levenson 1995; Visch et al. 2010), but very few attempts have been made to describe the complex experiences viewers may have of achieving a state of total immersion in a film.

The main research question for the present study is: How can we describe and measure obstacles to narrative immersion in a film experience designed to achieve total immersion? As part of this highly complex question and as a way to answer it, we have framed three sub-questions: A: What obstacles are there in a narrative experience, and how are they interrelated? B: Why do attentional distractors cause viewers to drop out of involvement at certain stages, but not at others? C: Are distractions affected by whether the viewing is taking place individually or in groups?

\section{Theoretical Background}

As there are different perspectives and descriptions of the term immersion, it is difficult to find a single unified definition that covers all aspects of immersion. There are already proposals for classifying different types of immersion (Ermi \& Mäyrä 2005; Ryan 2001), but each type is also linked to other perspectives within cognitive and emotional processes. Temporal immersion is described by Ryan (2001) as the suspense or information holdback of a certain narrative structure that triggers the viewer's curiosity through uncertain future events in the narrative construction. Similarly, as described by Bordwell (1985), it positions the viewer as an active participant. From a psychological and cognitive point of view, this can be explained using a mental model: the cognitive structures that symbolize different aspects of the surrounding world (Grodal 2009; Johnson-Laird 1983).

The sensation of emotional immersion is generally described as the level of emotional investment in a narrative that is experienced by the viewer (Ryan 2001). Similar concepts of empathy and sympathy are described from a psychological and philosophical perspective by Coplan (2004) in her characterization of the link between observers of fictional narratives and the characters in those narratives. This focus can also be found in transportation theory, where full engagement in a story is conceptualized as a distinct mental process, an integrative melding of attention, imagery and feelings (Green \& Brock 2000: 701). Transportation theory (Green \& Brock 2000) is conceptualized from a reading context, but also describes some fundamental conditions for media enjoyment, which comes from the experience of being immersed in a narrative world (Green, Brock \& Kaufman 2004).

Scholars have studied immersion in order to understand the implications of the story, plot, and the cognitive mechanisms the viewer interacts with when constructing and understanding the story (Bordwell 1985; Oatley 2002; Ryan 2001). In an audio-visual context, studies have shown how viewers' experience can be hindered (Busselle \& Bilandzic 2009) when narrative immersion competes with other mental processes for cognitive and emotional resources (Busselle \& Bilandzic 2008). Any external distractions that interrupt the narrative experience - such as noises, visual stimuli, and hunger - may have this effect, meaning that a negative component of narrative immersion is distraction (Busselle \& Bilandzic 2009). 


\section{The Wheel of Immersion}

For describing and exploring obstacles to narrative immersion in a film, we will propose a circular model (called the Wheel of Immersion) that focuses on the narrative experience and its subsequent story-related features. The basic tenet in the Wheel of Immersion is that viewers go through a dynamic progression of different levels of involvement by overcoming obstacles to narrative processing.

From a game perspective, Brown and Cairns (2004) discuss the idea of categorizing immersion into three levels of involvement: Engagement, Engrossment, and Total immersion. According to Brown and Cairns (2004), immersion is a degree of involvement with the media that moves along a path over time. The problem with this path is that it only shows the degree of involvement increasing, where each level is available if certain barriers are overcome. Moreover, Brown and Cairns' (2004) model lacks a description showing what happens when involvement is completely lost and how to recover the different levels again.

In the present study, we propose to alter Brown and Cairns' (2004) linear path of immersion over time and make it into a circular Wheel of Immersion that also includes a level of reality. The Wheel of Immersion is divided into four different levels - our own, Reality, and the levels from Brown and Cairns (2004): Engagement, Engrossment, and Total immersion. Brown and Cairns (2004) describe their understanding of immersion from a game perspective, whereas we transfer some of the concepts into an animation short film context. This is not unproblematic, of course, because these are two different media and different contexts. But the narrative experiences and the emotional and cognitive effects of playing computer games and watching films also have some great similarities. The most intense immersive narrative experience has been described across different media as transportation (Green \& Brock 2000), flow (Csikszentmihalyi 1990), presence (Lee 2004; Murray et al. 2007; Witmer \& Singer 1998), and total immersion (Brown \& Cairns 2004; Jennett et al. 2008). Essentially, these experiences have similarities regarding the users'/viewers' feeling of entering into a narrative world where it is possible to be fully absorbed in a story. Feelings of empathy and sympathy towards characters and the situations they are in are found across different media (Blanchet 2005; Coplan 2004; Hemenover \& Schimmack 2007; Smith 1995). Lee (2004) explains how lack of medium awareness is actually a defining element of an immersion-related experience.

The different levels in the Wheel of Immersion (Figure 1) represent how involved the viewer is with the medium and how aware he or she is of the surrounding reality. The different levels have associated arrows that point at other levels, representing what level the involved viewer typically can reach from his or her current level, or what level he or she can descend to if there are distractions. For example, when a viewer has reached the level of engagement, he or she can return to the level of reality or progress to engrossment. However, he or she cannot reach the level of total immersion directly, as the level of engrossment must be achieved first. Black lines have been placed between three of the levels to represent the obstacles the viewer must overcome in order to reach the next level. 
Figure 1. The Wheel of Immersion

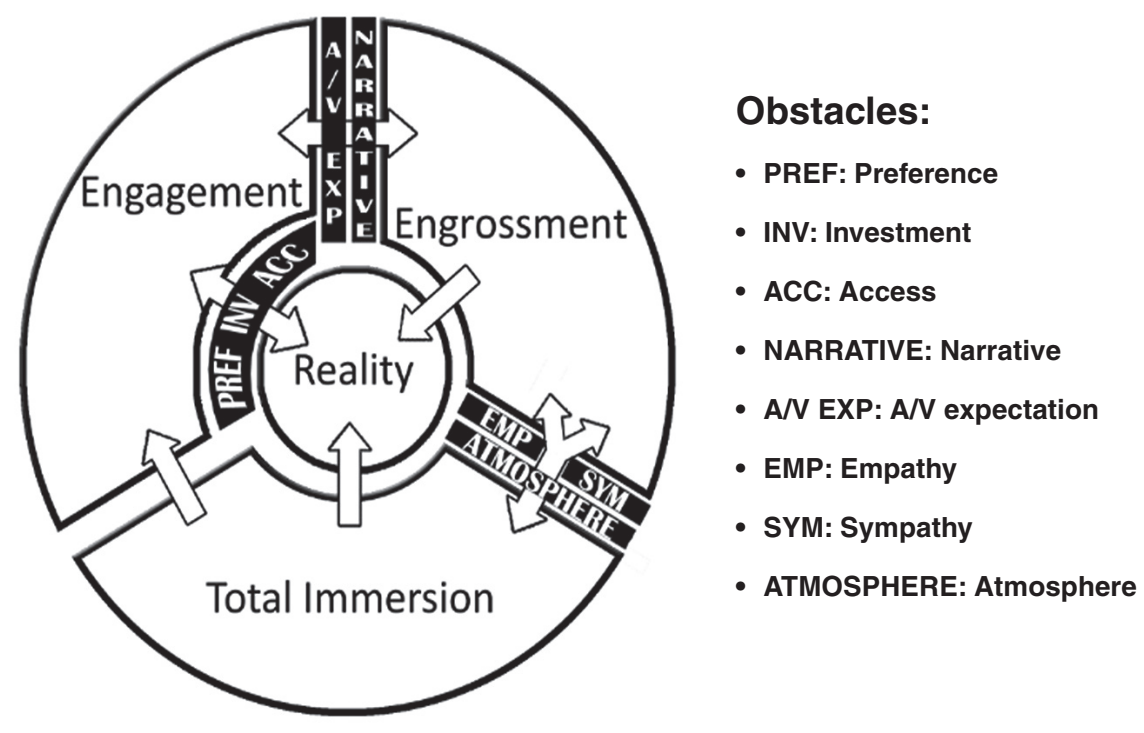

\section{From Reality to Engagement}

A viewer typically begins at the level of reality. The construct of reality in the context of audio-visual media is very complex and used in many different ways (Grodal 2002). We define reality as the level at which the viewer has total awareness of the surroundings and has no involvement with (or perceptual attention to) the film. This has also been described as nonengagement or disengagement (O'Brien \& Toms 2008) or what Tan (2008) labels as "the executive space". Busselle and Bilandzic (2009) use the term "the actual world". We agree with other scholars (Busselle \& Bilandzic 2009; Grodal 2002; Tan 2008) who suggest that it is easier for audience members to be involved in a story within a mediated world than in reality. From reality, the viewer has to overcome three human-dependent obstacles - access, investment and preference (Brown \& Cairns 2004) - in order to progress to engagement. Brown and Cairns (2004) use the term "barrier" to describe elements that have to be overcome before reaching another level, but they do not provide a theoretical explanation of this labelling. A barrier is normally a physical structure that blocks or impedes something. We use the term obstacle instead to describe both physical, cultural, and psychological/cognitive issues that lead to obstruction of the progression towards reaching a more intense immersion experience.

The obstacle of investment is the time and effort the viewer has to invest in the film in order to affect his or her attention and keep focused. Preference refers to the individual viewer's interest in the animation film and style, as the viewer will not keep focused if he or she finds it unappealing. For this reason, only a person who has, or develops, an interest in the film will be able to maintain his or her attention at a high enough level to overcome the obstacle. Access relies on the sensory feedback in which visuals and sound need to correspond in an appropriate manner. For example, access is difficult when the sound and visuals are unsynchronized in a film. 


\section{From Engagement to Engrossment}

Engagement is the level where the viewer is focused on the film with a low level of involvement yet still highly aware of his or her surroundings. Engrossment is where the viewer has total focus on the film with a high level of involvement. The viewer becomes less aware of the surroundings, knowing that they are there but finding them irrelevant and trying to ignore them. Referring to game construction, Brown and Cairns (2004) describe the barriers to proceeding from engagement to engrossment. It does not make sense to use the game construction label in this context. Instead we would like to propose two other obstacles to progressing to the level of engrossment: the narrative and Audio-Visual Expectations. The narrative obstacle is another term for narrative-driven immersion, which can be described as the "desire for the knowledge that awaits her at the end of narrative time" (Ryan 2001: 140). In other words, the narrative obstacle can be defined as suspense or information holdback of a certain narrative structure, similar to what is described within temporal immersion (Ryan 2001). The narrative obstacle implies that viewers' curiosity can be triggered through uncertain future events in the narrative (Bordwell \&Thompson 2010; Busselle \& Bilandzic 2008). The obstacle Audio-Visual Expectations is based on viewers' perceptions, which include both what is immediately perceived in a certain context and how the viewer can refer to prior experiences. These perceptual strategies have already been well described in different ways, e.g., in Grodal's PECMA flow theory $(1997 ; 2009)$ or Tan's (2008) two mental spaces (an executive space and an entertainment space).

\section{From Engrossment to Total Immersion}

Total immersion occurs when viewers are so involved that they detach themselves from the surroundings and lose their sense of time and place (Brown \& Cairns 2004; Jennett et al. 2008). Consequently, their full focus is on the animation film, which creates a feeling and thought of being present within the film. Brown and Cairns (2004) describe how empathy and atmosphere have to be overcome if one is to progress to the level of total immersion. We will use the same terms as Brown and Carins (2004), but put them in the context of film. Furthermore, below we propose to add an obstacle: sympathy. The empathy obstacle is inspired by the sensation of emotional immersion (Ryan 2001), meaning that it can be defined as a level of emotional attachment that the viewer has to the characters and the situations they are in. Empathy can be achieved because the human mind is able to simulate emotions, even though the source is fictional (Ryan 2001). When viewers empathize with a character, they take his or her psychological perspective and experience it in their imagination, to some degree, in order to simulate what the character is feeling (Blanchet 2005; Coplan 2004; Smith 1995). However, while empathizing with a character, viewers will still maintain a sense of separate identity (Coplan 2004). This means that even when one is deeply empathizing with what a character is experiencing, the separate sense of the self is never lost. Like empathy, the sympathy obstacle is inspired by the sensation of emotional immersion, and the level of emotional attachment the viewer has with the characters. However, instead of feeling with the characters, as happens in empathy, the viewer feels for the characters and the situations they are in (Blanchet 2005; Carroll 2001; Smith 1995). While sympathizing with the characters, one does not have to share the same experience (Coplan 2004). 
Sympathetic emotions are typically triggered by characters' emotions, which create the emotional response of feeling sorrow or concern for a distressed person, without feeling the same emotions (Coplan 2004). Lastly, sympathy can only be achieved when one has a cognitive understanding of the situation the characters are in, and a cognitive understanding of what the characters themselves are feeling in that situation (Blanchet 2005; Smith 1995).

Along with the aforementioned empathy and sympathy, atmosphere is the final obstacle that a viewer must overcome to reach total immersion. We will use the same definition as Brown and Cairns (2004), who define atmosphere as "the graphics, plot and sounds combin[ing] to create this feature" (Brown \& Cairns 2004: 1299), meaning that the combination of these elements is essential to creating the feature of atmosphere. But, inspired by Immanuel Kant (2007 [1793]), we propose to divide atmosphere into "beauty" and "sublimity". As described in $§ 16$, beauty "is immediately coupled with the representation through which the object is given (not through which it is thought)" (Kant 2007 [1793]), meaning that beauty relies on its form and representation and has to be considered a subjective judgment. The sensation of the sublime is connected with emotions, and Murdoch (1959) describes the difference between beauty and the sublime, based on Kant, in this way: "Whereas beauty results from a harmony between imagination and understanding, sublimity results from a conflict between imagination and reason" (Murdoch 1959: 44).

\section{Methods}

\section{A 3D Animation Short Film}

A 3D animation short film was made to test the Wheel of Immersion. Inspired by the narrative of the short film Paradox (Haccoun 2006), we developed a 3D animation short film using parts of the original soundscape from the Paradox film. The film is about two men trapped at the bottom of a well; the men appear to be from two different periods of time and are arguing about what is at the top of the well. We used Autodesk Maya 2010 and Adobe After Effects CS5 to create the 3D animation short film. The animation film is $15 \mathrm{~min}$ and $34 \mathrm{~s}$ in duration and includes two characters and two environments, which were modelled and animated to resemble Paradox (Haccoun 2006). The first environment represents the well and is where most of the dialogue takes place. The second environment represents the world surrounding the well, including sculpted and textured terrain, a skydome and animated clouds created from 3D fluids. The characters and camera movements in the animation film were created using a combination of key frame animation and graph editor in order to move the rigged characters around, creating a smooth transition between each of the motions. To ensure that the animation would follow the soundscape from the original film, a sound file-import was made to the timeline of Maya.

As well as the modelling and animation, some visual and technical effects were implemented to create a stronger atmosphere and A/V expectation. This included lowering the frame rate to achieve a more cartoon-like appearance, making a fog effect using fractal noise and implementing dust particles to create more atmosphere in the two environments. 
Figure 2. Frame from the $3 D$ Animation Short Film

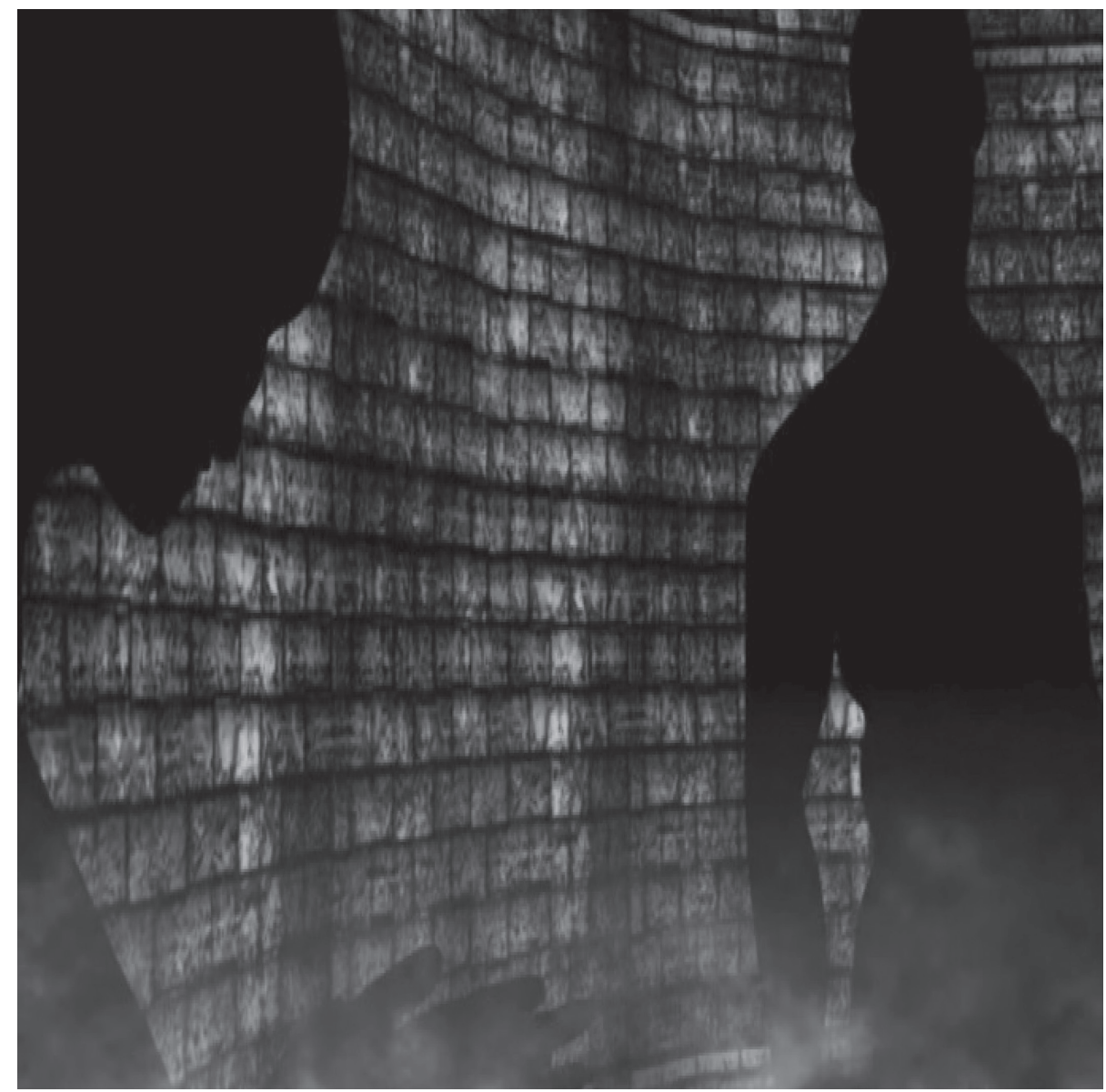

We chose to make the film in 3D for optimal perception and involvement, the aim being that some viewers could reach the level of total immersion. Using the $3 \mathrm{D}$ perspective (and non-physical nature), it was possible to give the film new spatial awareness. As Jones (2007) describes, the lack of physicality in the 3D perspective gives new omnipotent perceptions in relation to the ability to depict, engage and navigate cinematic space. Brown (2012: 264) also argues that the very nature of 3D makes a film even more immersive. We follow what most 3D CG features, namely focusing on what Power (2009) calls the naturalistic coding orientation with stylized 3D character models and high-profile dialogue actors.

\section{Test Design}

Setting up a good narrative immersion experiment is immensely difficult. In order to test which level of involvement viewers reached, and their progression through the Wheel of Immersion, we used a mixed method strategy combining an experiment (viewers watching the developed 3D animation film), an observation, a questionnaire 
and a structured interview. By employing mixed methods, we improved on previous attempts at measuring immersion based only on self-reporting questionnaires (Busselle \& Bilandzic 2009; Green \& Brock 2000; Visch et al. 2010) or on interviews (Brown \& Cairns 2004; O’Brien \& Toms 2008).

The final test included 38 subjects. Three additional pilot tests were conducted to eliminate presupposed information given during the test itself. The locations were arranged to provide minimum visual disturbance, meaning that posters, clocks, and unnecessary pieces of furniture were removed. Test participants were allowed secondary media, such as mobile phones, to distract them during the viewing. This was allowed because the aim was to provide as natural an environment as possible during the experience. However, this also created some uncontrollable variables.

Test subjects were chosen among university students between 19 and 28 years who had no prior knowledge of the study. The subjects were randomly assigned to one of four groups: Individuals With Distracters (IWD), n=10; Groups With Distracters (GWD), $\mathrm{n}=9$; Individuals Excluding Distracters (IED), $\mathrm{n}=10$; Groups Excluding Distracters (GED), $n=9$. To avoid rumours being spread about the test setup and use of visual distracters, each subject was instructed to remain silent about the test.

Figure 3. Test Setup

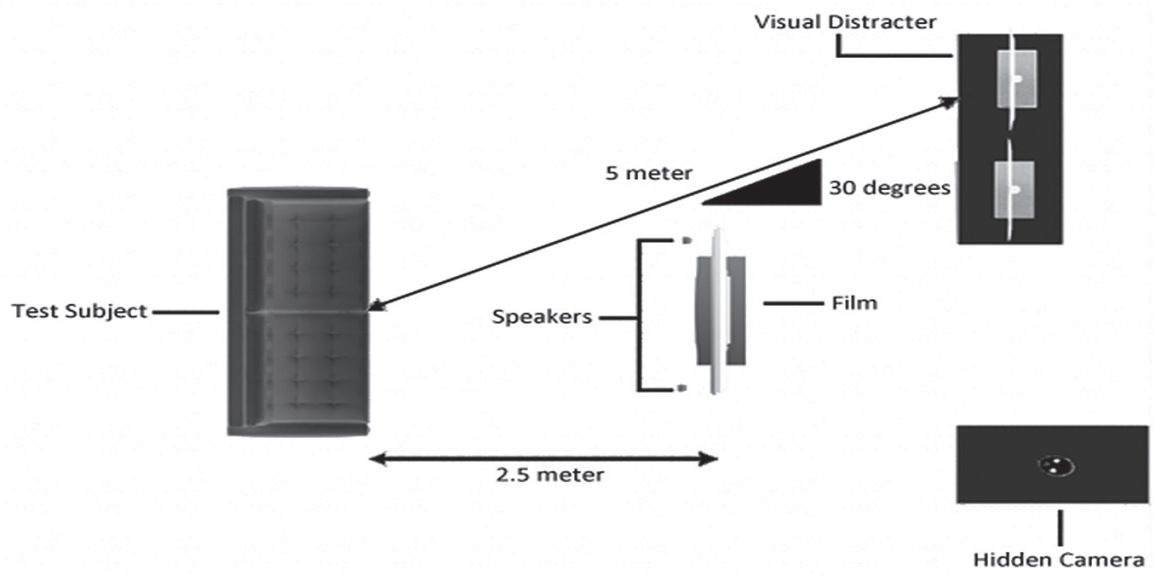

First, the subjects were briefly instructed to watch an animation short film. They were seated in a sofa and watched the animation on a 24 " television screen. The distance from the sofa to the television screen was 2.5 meters. No information was given concerning the film's content or visual distracters. In order to decrease the IWD and GWD groups' involvement, twelve black/white visual distracters were shown for 5 seconds (fade-in for 2 seconds, blank image for 1 second, and fade-out for 2 seconds), 5 meters from the test subjects (at a 30 degree angle). The visual distracters were displayed at the same time in the animation film for each subject/group with distractions. Furthermore a thirteenth colour image, together with a high frequency bell sound, was used in the ninth minute of the animation film. 


\section{Video Observation}

Video observation was used to capture viewers' emotional responses and see when they lost their attention while watching the 3D animation short film. We looked at interruptions in viewers' attention to determine how focused they were. The camera was hidden so the subjects were not aware that they were being observed. This was done to achieve the as natural a viewing situation as possible and to avoid the subjects becoming suspicious about the visual distracters or looking into the camera. For ethical reasons and to ensure good research practice, we informed the test subjects about the video observation in the debriefing (after the questionnaire and interview). They were all informed that the data obtained could only be kept with their permission. A framework of observational criteria was created for analysing the observed results, as illustrated in Table 1, below.

Table 1. Framework for Analysing Video Observations

\begin{tabular}{|l|l|}
\hline Observation & Evaluation \\
\hline $\begin{array}{l}\text { Quick eye movement beyond the screen } \\
\text { between } 0-1 \text { second }\end{array}$ & Low distraction \\
\hline $\begin{array}{l}\text { Longer eye focus beyond the screen be- } \\
\text { tween } 1-2 \text { seconds }\end{array}$ & Medium distraction \\
\hline $\begin{array}{l}\text { Quick head movement beyond the screen } \\
\text { between } 0-1 \text { second }\end{array}$ & Medium distraction \\
\hline $\begin{array}{l}\text { Longer eye and/or head movement beyond } \\
\text { the screen between } 1-2 \text { seconds or more }\end{array}$ & High distraction \\
\hline
\end{tabular}

\section{Questionnaire}

A questionnaire was used to measure self-reported levels of involvement; it consisted of 28 questions. We used a paper-and-pencil questionnaire, which was given to test subjects after they had watched the animation film. The questions were structured according to the progression in the Wheel of Immersion. The questionnaire was divided into eight sections, and the response alternatives were constructed using a five-point Likert scale.

Some of the specific questions for measuring level of involvement were inspired by the work of Busselle and Bilandzic (2009) and Green and Brock (2000). There are still limitations and problematic issues associated with questionnaire measures. As well as relying on participants' subjective opinions, they are also retrospective and rely on the subject's memory, which may only give incomplete reflections. This is why the questionnaire measurement should be combined with other methods.

\section{Interview}

In addition to the paper-and-pencil questionnaire, each subject participated in a brief individual interview. A structured interview (Bjørner 2015) was designed so that each interview consisted of exactly the same questions, in the same order. The purpose of the interview was to determine whether the subjects felt totally immersed at any time 
Table 2. The Questionnaire

\begin{tabular}{|c|c|c|c|c|c|}
\hline & Not at all & Slightly & Neutrally & Somehow & Yes fully \\
\hline \multicolumn{6}{|l|}{ 1. Investment } \\
\hline \multicolumn{6}{|l|}{$\begin{array}{l}1.1 \text { The animation film grabbed } \\
\text { my attention }\end{array}$} \\
\hline \multicolumn{6}{|l|}{ 2. Preference } \\
\hline \multicolumn{6}{|l|}{$\begin{array}{l}\text { 2.1 The animation film was } \\
\text { appealing }\end{array}$} \\
\hline \multicolumn{6}{|l|}{$\begin{array}{l}2.2 \text { I enjoyed the overall style of } \\
\text { the animation film }\end{array}$} \\
\hline \multicolumn{6}{|l|}{ 3. Access } \\
\hline \multicolumn{6}{|l|}{$\begin{array}{l}3.1 \text { I felt that the visuals and } \\
\text { sound matched each other }\end{array}$} \\
\hline \multicolumn{6}{|l|}{$\begin{array}{l}3.2 \text { I consider the sound and } \\
\text { visuals to be integrated with } \\
\text { the story }\end{array}$} \\
\hline \multicolumn{6}{|l|}{ 4. Narrative } \\
\hline \multicolumn{6}{|l|}{$\begin{array}{l}4.1 \text { While viewing, I frequently } \\
\text { received interesting cues from } \\
\text { the story }\end{array}$} \\
\hline \multicolumn{6}{|l|}{$\begin{array}{l}\text { 4.2 While viewing, I wanted to } \\
\text { know how the events would } \\
\text { unfold }\end{array}$} \\
\hline \multicolumn{6}{|l|}{$\begin{array}{l}4.3 \text { I wanted to learn how the } \\
\text { story ended }\end{array}$} \\
\hline \multicolumn{6}{|l|}{ 5. A/V Expectation } \\
\hline \multicolumn{6}{|l|}{$\begin{array}{l}\text { The scenery was consistently } \\
\text { logical and convincing }\end{array}$} \\
\hline \multicolumn{6}{|l|}{$\begin{array}{l}\text { The environment in the anima- } \\
\text { tion film is similar to the world } \\
\text { I live in }\end{array}$} \\
\hline \multicolumn{6}{|l|}{$\begin{array}{l}\text { The scenery evokes personal } \\
\text { memories }\end{array}$} \\
\hline \multicolumn{6}{|l|}{ 6.1 Empathy } \\
\hline \multicolumn{6}{|l|}{$\begin{array}{l}\text { At tense moments in the film, } \\
\text { I could feel the emotions the } \\
\text { characters felt }\end{array}$} \\
\hline \multicolumn{6}{|l|}{$\begin{array}{l}\text { During the film, I at certain } \\
\text { times lost my sense of self }\end{array}$} \\
\hline \multicolumn{6}{|l|}{$\begin{array}{l}\text { I never really shared the emo- } \\
\text { tions of the characters }\end{array}$} \\
\hline \multicolumn{6}{|l|}{ 6.2 Sympathy } \\
\hline \multicolumn{6}{|l|}{$\begin{array}{l}\text { I felt sorry for some of the } \\
\text { characters }\end{array}$} \\
\hline \multicolumn{6}{|l|}{$\begin{array}{l}\text { I recognized the emotional situ- } \\
\text { ation some of the characters } \\
\text { were in }\end{array}$} \\
\hline $\begin{array}{l}\text { I was concerned for some of } \\
\text { the characters }\end{array}$ & & & & & \\
\hline
\end{tabular}




\begin{tabular}{|c|c|c|c|c|c|}
\hline & $\begin{array}{c}\text { Very } \\
\text { unaffected }\end{array}$ & Unaffected & $\begin{array}{c}\text { Neither } \\
\text { unaffected } \\
\text { nor affected }\end{array}$ & Affected & $\begin{array}{l}\text { Very } \\
\text { affected }\end{array}$ \\
\hline \multicolumn{6}{|l|}{ 7.1. Atmosphere: Beauty } \\
\hline \multicolumn{6}{|l|}{$\begin{array}{l}\text { During the animation film how } \\
\text { affected were you by the follow- } \\
\text { ing atmospheric elements? }\end{array}$} \\
\hline \multicolumn{6}{|l|}{ Sound } \\
\hline \multicolumn{6}{|l|}{ Visuals } \\
\hline \multicolumn{6}{|l|}{ Overall narrative } \\
\hline & Not at all & Slightly & $\begin{array}{c}\text { Neither } \\
\text { unaffected } \\
\text { nor affected }\end{array}$ & Somehow & Yes fully \\
\hline \multicolumn{6}{|l|}{$\begin{array}{l}\text { 7.2. Atmosphere: The sub- } \\
\text { lime }\end{array}$} \\
\hline \multicolumn{6}{|l|}{$\begin{array}{l}\text { The atmosphere of the anima- } \\
\text { tion film evoked emotions in me }\end{array}$} \\
\hline \multicolumn{6}{|l|}{$\begin{array}{l}\text { While viewing, I had the sensa- } \\
\text { tion of something greater than } \\
\text { me }\end{array}$} \\
\hline \multicolumn{6}{|l|}{8.0 Distractors } \\
\hline \multicolumn{6}{|l|}{$\begin{array}{l}\text { I found my thoughts wandering } \\
\text { while watching the animation } \\
\text { film }\end{array}$} \\
\hline \multicolumn{6}{|l|}{$\begin{array}{l}\text { I had a hard time keeping my } \\
\text { focus on the film }\end{array}$} \\
\hline \multicolumn{6}{|l|}{ 9.0 Total immersion } \\
\hline \multicolumn{6}{|l|}{$\begin{array}{l}\text { During the animation film, I lost } \\
\text { track of time }\end{array}$} \\
\hline \multicolumn{6}{|l|}{$\begin{array}{l}\text { At times during the animation } \\
\text { film, I forgot that I was in the } \\
\text { middle of an experiment }\end{array}$} \\
\hline $\begin{array}{l}\text { During the animation film, my } \\
\text { body was in the room, but my } \\
\text { thoughts were inside the world } \\
\text { created by the story }\end{array}$ & & & & & \\
\hline
\end{tabular}

during the film. The interview consisted of the following three questions: (a) Did you notice the visual distracters? If yes, which ones? (This question was constructed to evaluate the degree to which the subjects felt interrupted by the imposed visual distracters) (b) Did you experience total immersion? (c) At which specific moments did you feel particularly immersed?

\section{Total Immersion}

By using the branches of the triangulated test setup and comparing the observational chart, questionnaire, and interview responses, we could see that Subject 7 (from the IWD group) did not progress far in the Wheel of Immersion and did not reach the level of total immersion. This is indicated in Figure 4, where the visual distracters and the subjects' distractions are displayed on a horizontal timeline representing the duration of the animation film (15 min, $34 \mathrm{~s}$ ). Above the timeline are the subjects' distractions 
throughout the film, while the visual distracters are displayed beneath the timeline. The distraction marked 'Reality' illustrates the points in time when the bell sound and image from the visual distracters are intended to force the subject out of the immersive level.

Figure 4. Frequency of Subject 7's Distractions, based on video observations

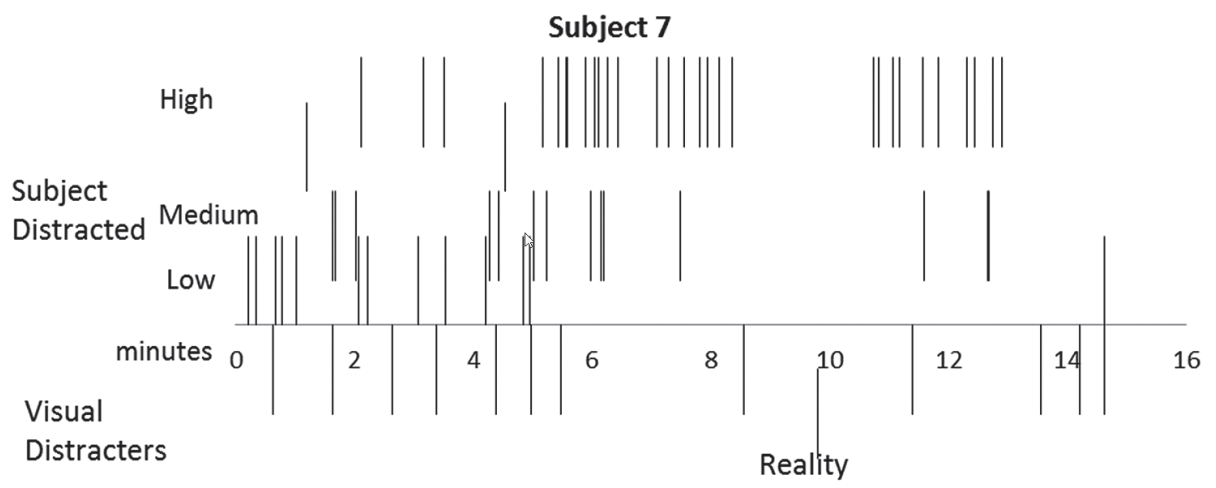

The observational chart (Figure 4) illustrates how Subject 7 was constantly distracted. The video observation indicates that Subject 7 even displays periods of time when the animation film was not the primary perceptual focus. The level of the distractions was compared with Subject 7's questionnaire responses and his interview statements, such as "This film did not capture my interest", and "I didn't feel immersed at any time during the film". In the questionnaire, Subject 7 responded "not at all" or "very unaffected" to almost all items on investment, preference, access, narrative, A/V expectation, empathy, sympathy and atmosphere (both beauty and the sublime), but "yes fully" to item 8.1 (I found my thoughts wandering while watching the animation film) and item 8.2 (I had a hard time keeping focus on the film). Elements indicating that Subject 7 was not totally disengaged the whole time can be seen in item 3.1 (I felt that the visuals and sound matched each other) and 3.2 (I felt that the sound and visuals were integrated with the story), where Subject 7 answered "somehow", which means that there was some attention to the film, at least to some of the overall visual and sound elements. We believe that the only reason Subject 7 stayed, invested time (remaining to the very end of the animation film) and put some focus on the film was because of the experimental set-up, which may have caused some feelings of obligation to stay. Based on findings from the mixed methods, Subject 7 is estimated to have barely reached the level of engrossment in the Wheel of Immersion. This indicates that Subject 7's level of immersion constantly alternated between the two basic levels of reality and engagement and that he had total awareness of his surroundings with no perceptual attention during most of the animation film. Subject 7 barely overcame the obstacle of investment, preference, whereas he to some extent overcame the access obstacle.

In the opposite end of the spectrum we find Subject 8 (also from the IWD group), as this subject reached the level of total immersion. Subject 8 was only distracted seven times during the film (see Figure 5), and all these distractions were measured as low: 
Figure 5. Frequency of Subject 8's Distractions, based on video observations

Subject 8

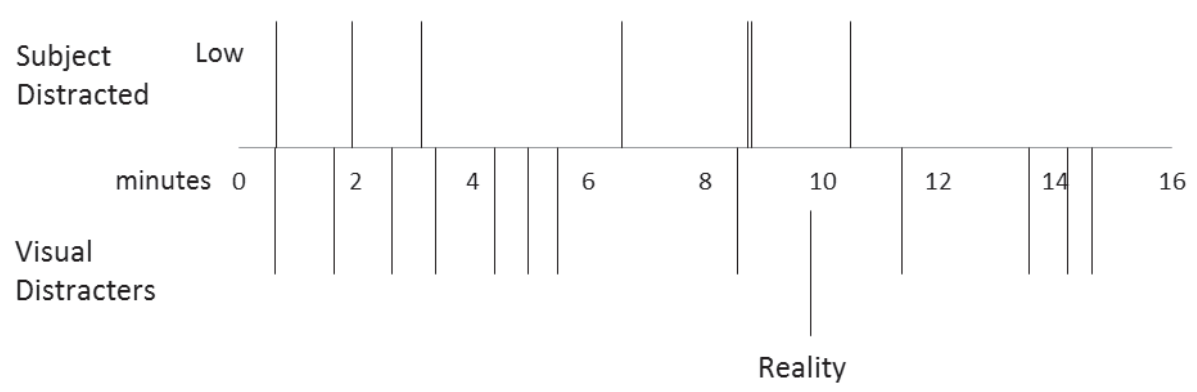

Triangulating the data, it appears that Subject 8 overcame all the obstacles and is estimated to have reached the level of total immersion several times during the animation film. The findings indicate that Subject 8 was in a mental process characterized by an integrated melding of attention, imagery and feelings (Green and Brock 2000), and identified with one character in the film, which was also stated during the interview. In the questionnaire, subject 8 answered "yes fully" to all items on investment, preference, access, narrative, emotions, sympathy and atmosphere: the sublime and total immersion. Subject 8 answered "slightly" to the items covering A/V Expectations, which might have something to do within the genre and the perception of what is immediately perceived in this specific (and rather artificial) viewing context, which may be related to subject 8 's brain mechanics. In response to both item 8.1 (I found my thoughts wandering while watching the animation film) and item 8.2 (I had a hard time keeping my focus on the film), Subject 8 answered "Not at all". This indicates that Subject 8 easily kept focus on the film, despite the distractions. In the interview, Subject 8 answered the question "Did you experience being fully immersed?" as follows: "I would say so, yes'. The findings also indicate that Subject 8 lost awareness of time and place, as he was not able to determine the duration of the film. In the interview, Subject 8 said: "Yes I completely recognize the sensation of forgetting time and place during the film".

\section{The Social Aspect and Progression}

When comparing the video observation of the different groups (both the frequency of the distractions and the social aspects of the subjects), two interesting observations can be made. The groups (GED and GWD) were either highly social, talking a lot to each other, or they respected maintaining a mutual silence, keeping their focus on the animation film. In other words, some groups were easily distracted by social activity, whereas other groups aimed to focus fully on the animation film. Group 4.2 showed almost no distractions at all, with only six instances of low distractions (eye movements beyond the screen between 0-1 second), whereas Group 3.2 was more distracted, with 18 low distractions, five medium distractions and five high distractions (which is still fewer than Subject 7).

The results from the groups (GED and GWD) were slightly different from what was anticipated. It was expected that the groups would show a much higher level of distractions due to the social aspect, but as the observations reveal (especially for Group 4.2), 
this was not the case. The results indicate that the level of distractions from watching the film with a small number of people (in this case three subjects) could have very different effects on the level of involvement in the film.

Of all subjects, it is estimated that 10 out of 38 achieved the level of total immersion. In the IWD group, 2 out of 10 indicated that they had reached total immersion, while one individual reached engrossment. In the IED group, four out of 10 reached total immersion, while one overcame the obstacles to reach engrossment. For the group test, two out of nine are estimated to have reached total immersion, while two subjects most likely reached engrossment. Finally, in the GED group, four out of nine reached total immersion, while only one subject reached the level of engrossment. In general, it was extremely difficult to determine exactly what level of involvement the test subjects reached, due partly to the very dynamic process of involvement during the animation film.

One interesting result is that subjects' distractions seem to appear in certain rhythms or waves. The data indicate that the subjects were more distracted in the beginning of the film, whereas they were less distracted in the end, which could be explained by the narrative obstacle in the Wheel of Immersion. This includes both the general plots and information holdback, or what Bordwell and Thompson described as the cause and effect, time and space (Bordwell \& Thompson 2010). Using Tan's terminology (2008), it is caused by the entertainment space being higher in the beginning and in the end, owing to the paradigm of scenarios with emotions.

Figure 6. Frequency of Subject 6's Distractions. Distractions appear in certain rhythms

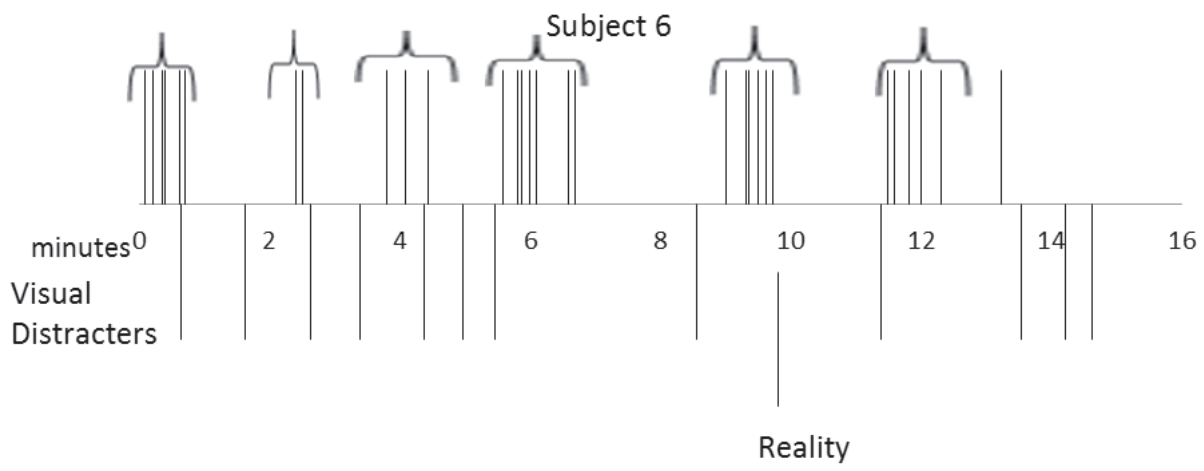

Figure 6 illustrates Subject 6's frequency of distractions during the animation film, showing how the distractions occur in certain rhythms. To illustrate how the wave structure of the visual distractions is repeated several times throughout the film experience, set brackets are placed around each cluster of distractions. Similar rhythms can be observed for almost all subjects, regardless of exposure or lack of exposure to distractions. The findings seem to indicate a higher concentration of distractions around the sixth minute of the animation film, which might have been caused by some perceptual and cognitive brain mechanism factors. But this pattern might also be a story-related matter, meaning simply that 'not much happens' in the sixth minute. As expected for the subjects exposed to distractions, many distractions occurred around the ninth minute, where the subjects were forced into a state of reality by the loud, frequent bell sound and coloured image. 


\section{Conclusions and Further Developments}

The aim of the present study was to describe and measure the complex experiences viewers' might have along the path to achieving total immersion in a $3 \mathrm{D}$ animation short film. The study focus was on the narrative experience and its subsequent story-related features. Clearly, trying to describe and measure the extent of involvement viewers might have while watching a narrative-driven media context is very complex indeed. This complexity is caused by the individual viewers' different narrative understandings, attentional focus and other perceptual brain mechanisms, which are at present difficult to measure in any precise manner. There are quite a number of different theoretical perspectives, in relation to both immersion and mental models perspectives in a media context. But there is still a need for greater clarity regarding viewers' involvement in narrative processing with further empirical data, also in the area of film watching. The present study has used a mixed method strategy that combines some rather traditional methods, but some supplementary data from a neuroscience paradigm may also be useful. We propose altering Brown and Cairns' (2004) immersive linear path of time into a circular model called the Wheel of Immersion. The circular model includes a level of reality, engagement, engrossment and total immersion; its purpose is to give a more dynamic understanding of how viewers progress to different levels of involvement by overcoming obstacles in narrative processing. The obstacles may be able to explain why attentional distractors cause viewers to drop out of immersion at certain stages, but not at others. A crucial point is actual time, which is often ignored as a factor of narrative experience. Further research is needed to investigate both the levels in the Wheel of Immersion, as well as the different obstacles.

The methodological design involved attempts to decrease viewers' involvement in the animation film by using distractions during the viewing. We observed that viewers reacted very differently to the distractions, in that some were very distracted and did not have the animation film as their primary perceptual focus. Other viewers kept their focus on the animation film seemingly without effort. Our study shows that viewers exposed to external distractions can reach total immersion, remaining focused on the film and having the sensation of forgetting time and place. In the present case involving a short film, the mental condition of high-focus watching could be maintained, despite experimental attemps to interrupt the narrative immersive experience.

The immersive progression was demonstrated by numerous subjects who showed similar rhythms of distractions, regardless of whether or not they had been exposed to distractions. For subjects in the distraction groups, we observed that they were more distracted in the ninth minute of the short film when they were forced into a state of reality by the loud, frequent bell sound and colour image. Our findings indicate that distractions were not dependent on whether the viewing was taking place individually or in groups. Some individual subjects were even more distracted than some of the subjects watching in groups, and some group viewing was highly focused on the film. In future research, it would be interesting to focus on these complex social group dynamics. Why were some of the groups tested either highly social, talking a lot to each other, while others were silent and focused on the film? 


\section{References}

Bjørner, Thomas (2015). 'Data collection'. In: Bjørner, Thomas (ed.) Qualitative methods for consumer research: The value of the qualitative approach in theory and practice. Copenhagen: Hans Reitzels Forlag.

Blanchet, Robert (2005). 'Deep impact: Emotion and performativity in contemporary blockbuster cinema'. In: Thomsen, Christiajn W. \& Krewani, Angela. (eds) Hollywood: Recent developments. Stuttgart: Axel Menges.

Bordwell, David (1985). Narration in the Fiction Film. Madison, WI: University of Wisconsin Press.

Bordwell, David \& Thompson, Kristin (2010) Film Art. An Introduction. $9^{\text {th }}$ ed. New York: McGraw-Hill.

Brown, William (2012) Avatar: Stereoscopic Cinema, Gaseous Perception and Darkness. Animation: An Interdisciplinary Journal 7(3): 259-271.

Brown, Emily \& Cairns, Paul (2004) 'A grounded investigation of game immersion'. Proceedings from the SIGCHI conference on Human factors in computing systems (CHI '04) (pp. 1297-1300). New York: ACM Press.

Busselle, Rick \& Bilandzic, Helena (2008) 'Fictionality and perceived realism in experiencing stories: A model of narrative comprehension and engagement'. Communication Theory 18(2): 255-280.

Busselle, Rick \& Bilandzic, Helena (2009) 'Measuring narrative engagement'. Media Psychology 12(4): 321-347.

Calleja, Gordon (2011) In-game: From Immersion to Incorporation. Cambridge: The MIT Press.

Carroll, Noël (2001) Beyond Aesthetics: Philosophical Essays. Cambridge: Cambridge University Press.

Coplan, Amy (2004) 'Empathic engagement with narrative fictions'. The Journal of Aesthetics and Art Criticism 62(2): 141-152.

Csikszentmihalyi, Mihaly (1990) Flow: The Psychology of Optimal Experience. New York: Harper Perennial.

Douglas, Jane Y. \& Hargadon, Andrew (2001) 'The pleasures of immersion and engagement: Schemas, scripts and the fifth business'. Digital Creativity 12(3): 153-166.

Ermi, Laura \& Mäyrä, Frans (2005) 'Fundamental components of the gameplay experience: Analysing immersion'. In: de Castell, Suzanne \& Jenson, Jennifer. (eds), Proceedings from the DiGRA conference: Changing views: Worlds in play (pp. 15-27). Vancouver, Canada.

Green, Melanie C. \& Brock, Timothy C. (2000) 'The role of transportation in the persuasiveness of public narratives'. Journal of Personality and Social Psychology 79(5): 701-721.

Green, Melanie C., Brock, Timothy C. \& Kaufman, Geoff (2004) 'Understanding media enjoyment: The role of transportation into narrative worlds'. Communication Theory 14(4): 311-327.

Grodal, Torben (1997) Moving Pictures. Oxford, UK: Clarendon Press.

Grodal, Torben (2002) 'The Experience of Realism in Audiovisual Representation'. In: Jerslev, Anne (eds) Realism and 'Reality' in Film and Media. Copenhagen: Museum Tusculanum Press.

Grodal, Torben (2009) Embodied Visions: Evolution, Emotion, Culture and Film. New York: Oxford University Press.

Gross, James J. \& Levenson, Robert W. (1995) 'Emotion elicitation using films'. Cognition and Emotion 9(1): 87-108.

Haccoun, Jeremy [Director] (2006). Paradox [Film]. USA: JH Productions.

Hemenover, Scott H. \& Schimmack, Ulrich (2007) 'That's disgusting!..., but very amusing: Mixed feelings of amusement and disgust'. Cognition and Emotion 21(5): 1102-1113.

Jennett, Charlene, Cox, Anna L., Cairns, Paul, Dhoparee, Samira, Epps, Andrew, Tijs, Tim \& Walton, Alison (2008) 'Measuring and defining the experience of immersion in games'. International Journal of HumanComputer Studies 66(9): 641-661.

Johnson-Laird, Philip N. (1983) Mental models. Cambridge: Cambridge University Press.

Jones, Mike (2007) 'Vanishing Point: Spatial Composition and the Virtual Camera'. Animation: An Interdisciplinary Journal 2(3):225-271.

Kant, Immanuel (2007 [1793]) Critique of Judgment. Translated by JC Meredith. Revised, edited and introduced by N Walker: Oxford University Press.

Lee, Kwan Min (2004) 'Presence explicated'. Communication Theory 14(1): 27-50.

Murdoch, Iris (1959) 'The Sublime and the Good'. Chicago Review 13(3): 42-55.

Murray, Craig D., Fox, Jesse \& Pettifer, Stephen (2007) 'Absorption, dissociation, locus of control and presence in virtual reality'. Computers in Human Behavior 23(3): 1347-1354.

Nedelcu, Marius (2013) 'Expanded Image Spaces. From Panoramic Image to Virtual Reality, Through Cinema'. Close Up: Film and Media Studies, 1(1): 44-53.

Oatley, Keith (2002) 'Emotions and the story worlds of fiction'. In: Green Melanie C., Strange Jeffrey J., \& Brock, Timothy C. (eds) Narrative Impact: Social and Cognitive Foundations. Mahwah, NJ: Lawrence Erlbaum, 39-70. 
O'Brien, Heather.L. \& Toms, Elaine G. (2008) 'What is user engagement? A conceptual framework for defining user engagement with technology'. Journal of the American society for Information Science and Technology 59(6): 938-955.

Power, Pat (2009) 'Animated expressions: Expressive style in 3D computer graphic narrative animation'. Animation: An Interdisciplinary Journal 4(2): 107-129.

Qin, Hua, Rau, Pei-Luen Patrick \& Salvendy, Gavriel (2009) 'Measuring player immersion in the computer game narrative'. International Journal of Human-Computer Interaction 25(2): 107-133.

Recuber, Tim (2007) 'The Rationalization and Reenchantment of Cinematic Space'. Space and culture, 10(3): 315-330.

Ryan, Marie-Laure (2001) Narrative as Virtual Reality: Immersion and Interactivity in Literature and Electronic Media. Baltimore, MD: Johns Hopkins University Press.

Smith, Murray (1995) Engaging Characters: Fiction, Emotion and the Cinema. Oxford: Clarendon Press.

Tan, Ed S. (1996) Emotion and the Structure of Narrative Film. Mahwah, NJ: Lawrence Erlbaum Associates, Inc.

Tan, Ed S. (2008) 'Entertainment is Emotion: The Functional Architecture of the Entertainment Experience'. Media Psychology 11(1): 28-51.

Visch, Valentine T., Tan Ed S. \& Molenaar, Dylan (2010) 'The emotional and cognitive effect of immersion in film viewing'. Cognition and Emotion 24(8): 1439-1445.

Witmer, Bob G. \& Singer, Michael J. (1998) 'Measuring presence in virtual environments: A presence questionnaire’. Teleoperators and Virtual Environments 7(3): 225-240.

THOMAS BJØRNER, Associate Professor, Aalborg University, Department of Architecture, Design and Media Technology, tbj@create.aau.dk.

ANDREAS MAGNUSSON, IT consultant, COMM2IG, duckdrew@gmail.com

ROBIN PASCAL NIELSEN, Web editor, IKEA Group, rp-Nielsen@hotmail.com 